\title{
Huntington disease-like syndrome due to c90RF72 expansions
}

INSERM

\section{Source}

INSERM. (1999). Orphanet: an online rare disease and orphan drug data base. Huntington disease-like syndrome due to C9ORF72 expansions. ORPHA:401901

Huntington disease-like syndrome due to C9ORF72 expansions is a rare, genetic neurodegenerative disease characterized by movement disorders, including dystonia, chorea, myoclonus, tremor and rigidity. Associated features are also cognitive and memory impairment, early psychiatric disturbances and behavioral problems. 